\title{
Cortical thickness in obsessive-compulsive disorder: multisite mega-analysis of 780 brain scans from six centres
}

Jean-Paul Fouche, Stefan du Plessis, Coenie Hattingh, Annerine Roos, Christine Lochner, Carles Soriano-Mas, Joao R. Sato, Takashi Nakamae, Seiji Nishida, Jun Soo Kwon, Wi Hoon Jung, David Mataix-Cols, Marcelo Q. Hoexter, Pino Alonso, OCD Brain Imaging Consortium,* Stella J. de Wit, Dick J. Veltman, Dan J. Stein and Odile A. van den Heuvel

\section{Background}

There is accumulating evidence for the role of fronto-striatal and associated circuits in obsessive-compulsive disorder (OCD) but limited and conflicting data on alterations in cortical thickness.

\section{Aims \\ To investigate alterations in cortical thickness and subcortical volume in $\mathrm{OCD}$.}

\section{Method}

In total, 412 patients with OCD and 368 healthy adults underwent magnetic resonance imaging scans. Betweengroup analysis of covariance of cortical thickness and subcortical volumes was performed and regression analyses undertaken.

\section{Results}

Significantly decreased cortical thickness was found in the OCD group compared with controls in the superior and inferior frontal, precentral, posterior cingulate, middle temporal, inferior parietal and precuneus gyri. There was also a group $\times$ age interaction in the parietal cortex, with increased thinning with age in the OCD group relative to controls.

\section{Conclusions}

Our findings are partially consistent with earlier work, suggesting that group differences in grey matter volume and cortical thickness could relate to the same underlying pathology of OCD. They partially support a frontostriatal model of OCD, but also suggest that limbic, temporal and parietal regions play a role in the pathophysiology of the disorder. The group $\times$ age interaction effects may be the result of altered neuroplasticity.

\section{Declaration of interest}

None.

\section{Copyright and usage}

(c) The Royal College of Psychiatrists 2017.
Obsessive-compulsive disorder (OCD) is characterised by intrusive thoughts (obsessions) and repetitive behaviours (compulsions). It is a fairly common mental disorder affecting $1-3 \%$ of the adult population over the course of a lifetime ${ }^{1}$ and it is associated with significant personal impairment and socioeconomic burden. ${ }^{2}$ Current models of OCD are based on animal laboratory work and human imaging studies, and emphasise the importance of frontostriatal networks. Meta-analyses of voxelbased morphometry (VBM) studies have found altered volumes in frontal and striatal regions, and in a broader range of structures, including parietal and limbic brain areas. ${ }^{3,4}$ A recent VBM mega-analysis on pooled raw magnetic resonance imaging (MRI) data from our multisite OCD Brain Imaging Consortium (OBIC), revealed significantly smaller volumes of frontal grey matter and white matter bilaterally, as well as group $\times$ age interactions in frontostriatal and limbic regions. ${ }^{5}$ Of particular interest in the meta-analyses and VBM mega-analysis is the finding of decreased grey matter volume in the dorsomedial prefrontal cortex, ${ }^{6,7}$ a region responsible for performance monitoring and emotional processing, processes which are affected in $\mathrm{OCD}^{8}$ Although we performed a VBM analysis on this data previously, other techniques such as surface-based methods can provide complementary information. Whereas VBM measures grey matter volume or density, surface-based methods such as FreeSurfer can calculate morphometric attributes in the native space of the participant, and allow a determination of cortical thickness.

*See online supplement DS1 for details of the members of the Consortium.
In addition, segmentation in VBM is suboptimal for some subcortical areas, such as pallidum and thalamus, because of the lack of clear grey-white contrast of these structures. FreeSurfer performs segmentation of whole subcortical structures and therefore it is not dependent on the contrast difference between tissue classes. ${ }^{10}$

Recently, OCD studies have been investigating surface-based measures such as cortical thickness. Studies have found decreased thickness in frontostriatal regions such as the orbitofrontal, inferior and middle frontal cortex, insula and anterior cingulate cortex. ${ }^{11-14}$ In addition, thinning in parietal and temporal regions was also evident in some studies. ${ }^{11,13,15}$ In contrast to these reports, some studies have reported greater thickness in OCD in regions of the right inferior frontal cortex, posterior middle temporal gyrus and right inferior parietal gyrus. ${ }^{16,17}$ Inconsistent cortical thickness findings in the literature can perhaps be attributed to the clinical heterogeneity of the samples. For example, in many of these studies the patients were already on medication and/or presented with comorbid depression. In smaller samples such as these, it is usually difficult to account for all possible confounders and therefore a mega-analysis is the ideal method to address these issues. The present study aimed to address the limitations of previous cortical thickness research on OCD, by pooling the structural MRI data from 412 patients with OCD and 368 healthy controls that were collected from six academic OCD centres across three continents (Asia, Europe and South America), participating in OBIC. ${ }^{5}$ This should provide sufficient sample size to potentially study smaller alterations in 
cortical thickness in a range of regions, and to examine the effects of clinical factors such as symptom severity, medication use and OCD symptom dimensions on cortical thickness and subcortical volumes, as well as relevant demographic considerations, such as between-group variance in age-related brain changes. Also, compared with meta-analyses, mega-analyses ensure the application of a common protocol, thus excluding the variability that different computing platforms or preprocessing strategies can introduce across studies. We hypothesised that consistent with previous VBM meta-analyses and other OCD cortical thickness studies, there would be cortical thinning in the frontal regions. Given the differences in segmentation between VBM and FreeSurfer, we also expected FreeSurfer to be more sensitive to volume changes in areas such as the putamen, pallidum and thalamus. ${ }^{3,4,7}$ Also, because of the variability in grey matter volume results between paediatric and adult patients with $\mathrm{OCD},{ }^{3,7}$ we expected to find group $\times$ age interactions in frontostriatal regions. Additionally, associations of cortical thickness changes with OCD severity and symptom dimensions were expected as previous studies have shown that Yale-Brown Obsessive-Compulsive Scale $(\mathrm{YBOCS})^{18}$ and symptom dimensions correlate with frontal and parietal thickness in patients with OCD. ${ }^{12,14,15}$ Finally, given the significant variability in previous cortical thickness studies, potentially as a result of comorbid anxiety/depressive disorders as well as medication use, additional analyses were performed, excluding patients on medication and with lifetime comorbid anxiety or depression.

\section{Method}

\section{Participants and data processing}

Data were obtained from six sites as part of the OBIC. The cohort has been described in detail previously. ${ }^{5}$ Participants were screened for DSM-IV Axis I disorders ${ }^{19}$ and exclusion criteria were aged under 18 and above 65, current psychotic disorder, recent history of a substance use disorder, intellectual disability and severe organic or neurological pathology. Other psychiatric comorbidity was allowed if OCD was the primary diagnosis. The complete sample consisted of 412 patients with OCD (the OCD group) and 368 healthy controls (control group).

The collected $780 \quad 1.5$ Tesla $T_{1}$-weighted MRI scans were transferred and processed with FreeSurfer V4.5 (http:// surfer.nmr.mgh.harvard.edu) on the Nehalem cluster at the Centre for High Performance Computing (CHPC), Rosebank, Cape Town, South Africa. The standard processing pipeline known as recon-all has been described and validated previously. ${ }^{20}$ In short, $T_{1}$-weighted images were normalised, bias-field corrected and skull-stripped. Inner and outer cortical surfaces were modelled as triangular tessellation. Cortical thickness measurements were obtained by calculating the distance (in $\mathrm{mm}$ ) between pial and grey-white matter surfaces at each vertex location. ${ }^{20}$ These surfaces were then normalised to the 'fsaverage' template included with FreeSurfer by using a curvature matching technique, allowing vertex-wise comparison of cortical thickness across participants. ${ }^{9}$ In addition, data were smoothed with a Gaussian kernel of $10 \mathrm{~mm}$ full-width at half maximum. For subcortical structures, the brain was segmented into volume-based labels utilising probabilistic methods. After reconstruction, each individual scan was visually inspected for large errors in segmentation and manually corrected if necessary.

Subcortical regions were parcellated in FreeSurfer to obtain volumes for all the major subcortical regions, namely hippocampus, amygdala, putamen, pallidum, caudate, thalamus and nucleus accumbens for each hemisphere. Total intracranial volume was extracted from FreeSurfer and used for brain size correction by utilising the proportion method for each of the subcortical regions. ${ }^{21}$

\section{Statistical analysis}

Vertex-wise analyses were performed on cortical thickness data with FreeSurfer's mri glmfit software. Subcortical mean volumes were imported into SPSS 20.0. For both the cortical thickness and subcortical volumes, group effects were investigated by utilising a general linear model (GLM) with age, gender, scan sequence and level of education as covariates. In addition, post hoc analyses were performed to determine whether there was an interaction of scan sequence with main group effects between the control and OCD groups (see online supplement DS2 and online Tables DS1-3). To investigate the effects of age and group $\times$ age interactions, we used both linear and quadratic models of age as regressors in the analysis. In addition to the main group comparison and the group $\times$ age interaction, the neural correlates of clinical variability within the patient group were assessed. Multiple regression analyses were performed to investigate the effects of OCD severity and symptom dimensions within the OCD group. The analysis was also repeated after excluding (a) patients with OCD on medication, (b) patients with comorbid anxiety and (c) patients with comorbid depression in turn (see Results and online supplement DS3 and online Tables DS4-7). In addition, within the OCD group the influence of medication status and the presence of lifetime anxiety disorder(s) or major depressive disorder on cortical thickness and subcortical volumes were studied (see online supplements DS4-7 and online Tables DS8-10 for details on these analyses).

Data-sets for these regression analyses were selected based on the availability of clinical information. Covariates of no interest included age, gender, sequence and level of education, as well as total YBOCS ${ }^{18}$ score as indication of illness severity in the case of the symptom dimension analysis. All cortical thickness analyses were corrected with 10000 Monte-Carlo simulations $(P<0.05)$ using Freesurfer's mri_glmfit software. SPSS subcortical analyses were Bonferroni corrected with an $\alpha$ of $P<0.0036$ for the 14 regions-of-interest ( 7 regions per hemisphere) when examining main group effects.

In order to confirm whether group differences were not confounded by group differences in age, gender, education and ethnicity, the analysis was repeated in a smaller sample group that were matched for these attributes (see de Wit et $a l^{5}$ for further details on how these groups were matched).

\section{Results}

\section{Sample characteristics}

The OCD $(n=412)$ and control $(n=368)$ groups were matched on gender, handedness and ethnicity. However, patients were significantly older (OCD group 32.1 years (s.d.=9.6); control group 30.2 years (s.d. 9.3), $t=2.9, P=0.004$ ) and had a lower level of education (OCD group 13.7 years (s.d. $=2.8$ ); control group 14.6 years (s.d. $=3.1$ ) $, t=-4.0, P<0.001$ ) than the control group. The OCD group had a mean YBOCS score of 24.9 (s.d. $=6.2$ ) and a mean age of clinical onset of 20.1 years (s.d. $=8.7$ ). See de Wit et $a l,{ }^{5}$ online supplement DS2 and online Table DS11 for further details on the demographic and clinical characteristics of the total sample, as well as the age-, gender, education and ethnicitymatched sample ( $n=329$ patients with OCD and $n=316$ healthy controls). 


\section{Group comparisons}

The cortical thickness in the OCD group compared with healthy controls was significantly decreased in the superior (left hemisphere $z=5.515, \quad P<0.0001$; right hemisphere: $z=2.368$, $P=0.0179$ ) and inferior frontal (right hemisphere: $z=3.060$, $\mathrm{p}=0.0022$ ), precentral (right hemisphere: $z=3.354, P=0.0008$ ), posterior cingulate (left hemisphere: $z=3.288, P=0.0010$ ), middle temporal (right hemisphere: $z=3.959, P=0.0001$ ), inferior parietal (left hemisphere: $z=4.259, P<0.0001$ ) and precuneus gyri (right hemisphere: $z=3.781, P=0.0002$ ) (see Table 1 and Fig. 1). The OCD group, compared with the control group, also showed decreased volume of the hippocampus (left hemisphere: $F=8.630, P=0.0002$; right hemisphere: $F=6.727, P=0.0007)$ (Table 2). After performing a post hoc analysis in the subsample matched for age, gender, educational level and ethnicity, similar findings were obtained (see online Tables DS1 and DS2). There was also no significant sequence by diagnosis interaction for the group comparisons (online supplement DS2). There were no regions that showed significantly increased cortical thickness or subcortical volume in the OCD group when compared with healthy controls.

\section{Group $\times$ interaction effects}

There were no significant group $\times$ age interaction effects for cortical thickness, nor were there significant group $\times$ age effects for the subcortical volumes. However, the post hoc matched sample analysis showed that compared with controls, the OCD group have significant age-related cortical thinning of the left inferior parietal gyrus (linear: $z=2.957, P=0.0031$, quadratic: $z=3.197, P=0.0014)$, in addition to quadratic age-related cortical thinning of the right superior parietal gyrus $(z=2.355, P=0.0185)$ (see online Table DS3).

\section{Association and exclusion of clinical variables}

Disease severity

There was an association between increased disease severity (YBOCS score) and decreased cortical thickness in the bilateral lateral occipital gyrus (left hemisphere: $z=-5.634, P<0.0001$; right hemisphere: $z=-3.984, P=0.0001$, see online Table DS12).

\section{Comparison between controls and participants}

in the OCD group not on psychotropic medication

Unmedicated participants in the OCD group $(n=222)$ compared with healthy controls $(n=368)$ showed decreased cortical thickness in the left superior frontal $(z=4.222, P<0.0001)$, right inferior frontal $(z=5.680, \quad P<0.0001)$, right precentral $(z=3.771, \quad P=0.0002)$, right posterior cingulate $(z=5.639$, $P<0.0001$ ) and bilateral middle temporal (left hemisphere: $z=6.470, P<0.0001$; right hemisphere: $z=5.840, P<0.0001)$ gyri (online Table DS4). ANCOVA analyses of subcortical volumes revealed no significant differences between the unmedicated participants in the OCD group and healthy controls.

\begin{tabular}{|c|c|c|c|c|c|}
\hline \multirow[b]{2}{*}{ Grey matter region and hemisphere } & \multicolumn{2}{|c|}{ Thickness, mm: mean (s.d.) } & \multirow{2}{*}{$\begin{array}{l}\text { Local maxima } \\
\text { Z-value }\end{array}$} & \multirow[b]{2}{*}{$P$} & \multirow{2}{*}{$\begin{array}{c}\text { Talaraich coordinates } \\
\qquad(x, y, z)\end{array}$} \\
\hline & Control group & OCD group & & & \\
\hline \multicolumn{6}{|l|}{ Frontal } \\
\hline \multicolumn{6}{|l|}{ Superior frontal } \\
\hline Left & $3.1093(0.2089)$ & $3.0545(0.2258)$ & -5.515 & $<0.0001$ & $-8.8,26.6,32.7$ \\
\hline Right & $2.9013(0.2009)$ & $2.8609(0.2049)$ & -2.368 & 0.0179 & $9.1,36.1,26.8$ \\
\hline Inferior frontal, right & $2.7068(0.1697)$ & $2.6593(0.1714)$ & -3.060 & 0.0022 & $50.1,23.8,16.3$ \\
\hline Precentral right & $2.6220(0.1673)$ & $2.5760(0.1790)$ & -3.354 & 0.0008 & $47.6,5.2,14.8$ \\
\hline \multicolumn{6}{|l|}{ Parietal } \\
\hline Inferior parietal, left & $2.4988(0.1637)$ & $2.4504(0.1639)$ & -4.259 & $<0.0001$ & $-37.3,-57.1,26.2$ \\
\hline Posterior cingulate, left & $2.5745(0.1693)$ & $2.5154(0.1736)$ & -3.288 & 0.0010 & $-5.9,-14.1,36.7$ \\
\hline Precuneus, right & $2.6424(0.2421)$ & $2.5751(0.2395)$ & -3.781 & 0.0002 & $7.0,-56.0,17.8$ \\
\hline \multicolumn{6}{|l|}{ Temporal } \\
\hline Middle temporal, right & $2.7882(0.1645)$ & $2.7292(0.1651)$ & -3.959 & 0.0001 & $57.9,-42.7,-8.5$ \\
\hline
\end{tabular}

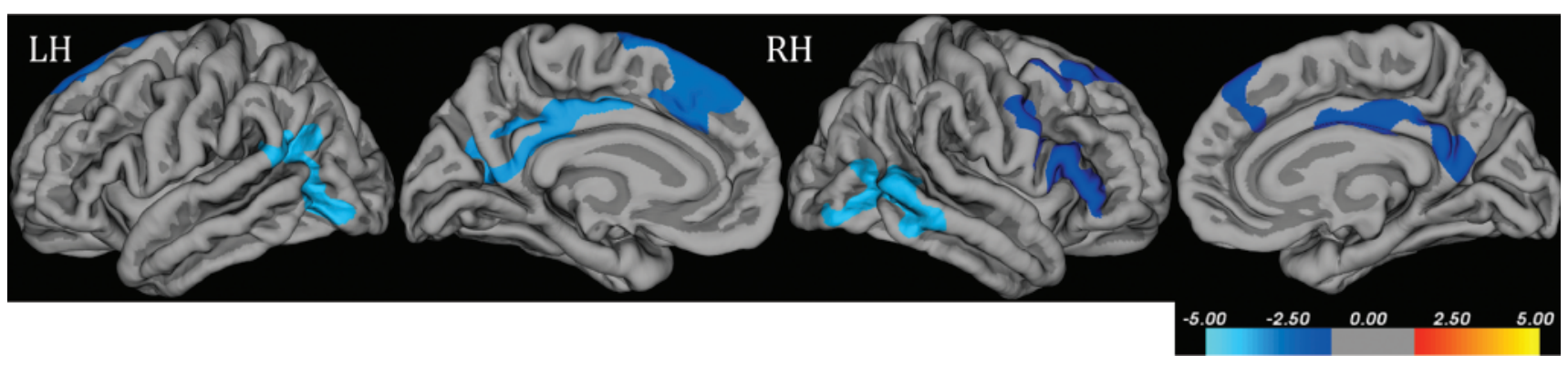

Fig. 1 Grey matter regions exhibiting decreases in cortical thickness for the obsessive-compulsive disorder group compared with the control group with analysis of covariance.

Results are shown at $P<0.05$ corrected for multiple comparisons with Monte-Carlo simulation. Covariates of no interest included scan sequence, level of education, age and gender. The colour bar indicates the $z$-value at each vertex. LH, left hemisphere; RH, right hemisphere. 


\begin{tabular}{|c|c|c|c|c|}
\hline \multirow[b]{2}{*}{ Subcortical region and hemisphere } & \multicolumn{2}{|c|}{ Volume, $\mathrm{mm}^{3}$ : mean (s.d.) } & \multirow[b]{2}{*}{$F$-value } & \multirow[b]{2}{*}{$P$} \\
\hline & Control group & OCD group & & \\
\hline Basal ganglia total volume, left+right & $38087.3(3984.2)$ & $38014.5(3986.5)$ & 0.093 & 0.761 \\
\hline \multicolumn{5}{|l|}{ Thalamus } \\
\hline Left & 7361.8 (633.2) & $7368.8(642.7)$ & 0.269 & 0.043 \\
\hline Right & $7348.1(544.9)$ & $7309.4(553.1)$ & 2.093 & 0.011 \\
\hline \multicolumn{5}{|l|}{ Caudate } \\
\hline Left & $3637.3(371.2)$ & $3662.4(376.7)$ & 0.418 & 0.037 \\
\hline Right & $3653.4(387.4)$ & $3666.6(393.2)$ & 0.031 & 0.061 \\
\hline \multicolumn{5}{|l|}{ Putamen } \\
\hline Left & $5841.7(528.6)$ & 5855.5 (536.5) & 0.134 & 0.051 \\
\hline Right & $5569.9(497.8)$ & $5615.2(505.2)$ & 0.044 & 0.060 \\
\hline \multicolumn{5}{|l|}{ Pallidum } \\
\hline Left & $1740.8(186.3)$ & $1769.0(189.1)$ & 2.174 & 0.010 \\
\hline Right & $1609.7(203.7)$ & $1644.6(206.7)$ & 3.178 & 0.005 \\
\hline \multicolumn{5}{|l|}{ Hippocampus } \\
\hline Left & $4270.1(361.2)$ & $4205.2(366.6)$ & 8.630 & $0.0002^{* *}$ \\
\hline Right & $4357.6(354.9)$ & $4300.0(360.2)$ & 6.727 & $0.0007^{*}$ \\
\hline \multicolumn{5}{|l|}{ Amygdala } \\
\hline Left & $1517.6(159.1)$ & $1524.3(161.6)$ & 0.029 & 0.061 \\
\hline Right & $1598.0(172.9)$ & $1594.2(175.4)$ & 0.371 & 0.039 \\
\hline \multicolumn{5}{|l|}{ Accumbens-area } \\
\hline Left & $597.4(95.0)$ & $591.4(96.4)$ & 0.734 & 0.028 \\
\hline Right & $600.6(91.6)$ & 590.0 (92.9) & 1.918 & 0.012 \\
\hline
\end{tabular}

Comparison between controls and participants in the OCD group without lifetime comorbid anxiety

Participants in the OCD group without lifetime comorbid anxiety $(n=190)$ compared with healthy controls $(n=368)$ demonstrated decreased cortical thickness in the right insula $(z=8.205$, $P<0.0001)$, right caudal middle frontal $(z=3.530, P=0.0004)$, left paracentral $(z=10.419, \quad P<0.0001)$ and right pericalcarine $(z=12.531, P<0.0001)$ gyri (online Table DS5). There was also decreased volume of the bilateral hippocampus (left hemisphere: $F=7.677, P<0.001$; right hemisphere: $F=5.057, P=0.002)$ in the OCD group compared with healthy controls (online Table DS7).

\section{Comparison between controls and OCD group}

without lifetime comorbid depression

Patients without lifetime comorbid depression $(n=287)$ compared with healthy controls $(n=368)$ showed decreased cortical thickness in the left superior frontal $(z=4.576, P<0.0001)$, bilateral inferior frontal (left hemisphere: $z=2.869, P=0.0041$; right hemisphere: $z=3.516, \quad P<0.0004)$, left rostral middle frontal $(z=2.823$, $P=0.0048)$, right medial orbitofrontal $(z=3.240, P=0.0012)$ and left lateral occipital $(z=4.115, P<0.0001)$ gyri (online Table DS6). ANCOVA analyses of subcortical volumes revealed no significant differences between the OCD group without depression and healthy controls.

\section{Symptom dimensions}

Information on the presence/absence of specific OCD symptom dimensions was available for 331 patients. The presence of five symptom dimensions was assessed. These included the aggressive/checking, contamination/cleaning, symmetry/ordering, sexual/religious obsessions and hoarding subdimensions. A dimension was considered as 'present' if the patient has reported a current or lifetime history of at least one of the symptoms. For an overview of the results of the correlation analyses on symptom dimensions, see online supplement DS8 and Table DS12. The contamination/cleaning dimension was associated with increased cortical thickness in the left lateral orbitofrontal $(z=5.840$, $P<0.0001)$, precentral $(z=4.109, P<0.0001)$ and right frontal gyri $(z=5.275, P<0.0001)$. The hoarding dimension was associated with increased cortical thickness in the bilateral inferior frontal gyri (left hemisphere: $z=4.543, P<0.0001$; right hemisphere: $z=4.965$, $P<0.0001)$, left lateral orbitofrontal $(z=3.062, P=0.0022)$, superior parietal $(z=2.417, P=0.0156)$, middle temporal $(z=3.256$, $P=0.0011)$ and lateral occipital gyri $(z=3.501, P=0.0005)$, right superior frontal $(z=6.088, P<0.0001)$ and medial orbitofrontal gyri $(z=4.012, P=0.0001)$ and cuneus $(z=2.323, P=0.0202)$. The sexual/religious dimension was associated with increased cortical thickness in the left isthmus cingulate $(z=4.802$, $P<0.0001)$, rostral middle frontal $(z=4.383, P<0.0001)$, lateral occipital $(z=4.035, \quad P=0.0001)$, right lateral orbitofrontal $(z=2.558, P=0.0105)$, superior parietal $(z=4.237, P<0.0001)$, supramarginal gyri $(z=3.183, P=0.0015)$ and precuneus $(z=2.959, \quad P=0.0031)$. The symmetry/order dimension was associated with increased cortical thickness in left insular $(z=5.263, P<0.0001)$, lingual $(z=3.040, P=0.0024)$, precentral $(z=5.444, \quad P<0.0001)$ and postcentral gyri $(z=3.822$, $P=0.0001)$, as well as right medial orbitofrontal $(z=3.594$, $P=0.0003)$ and lateral occipital gyri $(z=3.729, P=0.0002)$. It was also associated with decreased cortical thickness in the right superior temporal gyrus $(z=-3.927, P=0.0001)$. The aggression/ checking dimension was associated with increased cortical thickness in the right lateral occipital gyrus $(z=2.924$, $P=0.0035)$, as well as lower entorhinal thickness $(z=-3.930$, $P=0.0001)$. There were no significant positive or negative association with subcortical volumes in the OCD group.

\section{Discussion}

\section{Main findings}

This FreeSurfer mega-analysis of multisite $T_{1}$-weighted MRI scans showed that patients with OCD exhibit decreased cortical 
thickness in a number of frontoparietal regions. These included the superior and inferior frontal gyri findings similar to previous VBM meta-analyses and cortical thickness work. In addition to other temporoparietal regions such as the precentral, posterior cingulate, middle temporal, inferior parietal and precuneus gyri. There was also limbic involvement as evidenced by decreased hippocampal volume compared with healthy controls. Another finding was a group $\times$ age interaction effect for left parietal thickness suggesting that there is exaggerated cortical thinning of the parietal gyri with advancing age and/or illness duration in patients compared with controls.

The most notable finding in this FreeSurfer study, consistent with the findings of our VBM mega-analysis, ${ }^{5}$ is the involvement of the inferior frontal gyrus/operculum and superior frontal gyrus/dorsomedial prefrontal cortex in OCD. This is important, insofar as findings from smaller studies have been contradictory. ${ }^{22,23}$ This finding in both the mega-analyses suggests that cortical thinning and grey matter volume could relate to a similar underlying pathological process responsible for abnormalities in these regions. This finding was even more pronounced when excluding patients on medication and with lifetime comorbid anxiety and depression, indicating that it may be specific to OCD. Cortical thinning in the inferior frontal gyrus and superior frontal gyrus is also consistent with the findings of some previous VBM studies ${ }^{7,22,24}$ and one cortical thickness study. ${ }^{11}$ Decreased cortical thickness of the inferior frontal gyrus and superior frontal gyrus may be involved in impairments of cognitive and emotional control in OCD; the inferior frontal gyrus together with the anterior insula is part of a circuit implicated in behavioural inhibition and disgust-related responses. ${ }^{8}$ The inferior frontal gyrus is also part of a network implicated in working memory and emotion regulation, ${ }^{8}$ together with the interconnected precentral and superior (including dorsomedial) frontal gyri, that both show decreased cortical thickness in the present study. The decreased thickness in these regions could also be related to the abnormal connectivity evident in the default-mode and attention network of people with OCD. Previous investigations have demonstrated that there is an increased functional connectivity between the prefrontal and insula/cingulate regions, suggesting greater cognitive control, but weaker connectivity between precentral gyrus and prefrontal cortex (suggesting lower motor inhibition) in OCD. ${ }^{25}$ This incongruence between the dorsal and ventral networks has also been demonstrated in another study which investigated the structural covariance of cortical thickness networks in OCD. ${ }^{26}$

Both our data and previous work suggest at least some involvement of parietal and temporal regions in OCD, ${ }^{4,7,11}$ consistent with the suggested involvement of the fronto-parietal and limbic circuits in the pathophysiology of OCD. The results also held true after exclusion of patients on medication and with lifetime comorbid anxiety and/or depression. Evidence from functional MRI and cognitive testing has demonstrated that parietal and temporal regions are involved in impairment of inhibitory control ${ }^{27,28}$ and executive planning ${ }^{29,30}$ in OCD, whereas the hippocampus together with amygdala and anterior cingulate cortex may comprise affective components of the OCD fronto-cortical circuit. ${ }^{31}$ Resting-state studies confirm abnormal connectivity of frontoparietal networks, with increased connectivity of motor networks, ${ }^{32}$ but decreased connectivity in frontotemporal networks, especially in the posterior cingulate (an important hub of the default-mode network)..$^{33,34}$

Although previous VBM studies have found greater striatal volume, ${ }^{4,7}$ the same findings could not be replicated in this mega-analysis. A possible reason for the absence of this finding is as a result of the manner in which Freesurfer segments subcortical structures. Whereas VBM performs segmentation of the striatum based on contrast differences between grey and white matter, Freesurfer segments whole structures based on probabilistic information from a predefined atlas. ${ }^{35}$ Therefore, only global changes in subcortical structure can be inferred from Freesurfer, rather than local morphology as with a voxel-based method such as VBM. It is thus possible that these volume increases in the striatum are not detectable when averaging across the whole structure. Also, because the MRI scans were $1.5 \mathrm{~T}$, there are limitations to the signal-to-noise ratio of the data, as well as to the resolution. ${ }^{36}$

\section{Findings from the group $\times$ age interaction analyses}

Group $\times$ age interaction analyses show that the inferior and superior parietal regions demonstrate relatively exaggerated age-related cortical thinning in the OCD group compared with controls. Cortical thinning with age is normal, ${ }^{37,38}$ but this exaggerated decrease in the parietal lobes of our patient cohort might explain some characteristics typical of OCD: the parietal lobes are involved in planning and response inhibition, both processes that are impaired in OCD and potentially aggravated with age and disease duration. ${ }^{31}$ Although we did not find any significant association of disease severity or disease duration with grey matter volume $^{5}$ or thickness, the possibility exists that the more pronounced thinning in parietal regions in older patients, compared with older controls, may partly be explained by longer disease duration. Additionally the association with age was squared, indicating that rapid parietal cortical thinning occurred at set age intervals, rather than linear progressive thinning as patients became older. Even in healthy individuals, grey matter density decline in parietal regions is non-linear, with the majority of loss occurring between 50 and 70 years of age. ${ }^{37}$ To further disentangle the effect of ageing and the effect of disease chronicity a longitudinal design is needed.

\section{Comparison with findings from other VBM studies}

There is some consistency of this study with previous VBM metaanalyses. Most notably previous VBM studies have also found grey matter volume decreases in inferior frontal, orbitofrontal and dorsolateral prefrontal regions. ${ }^{4,22}$ However in contrast to previous VBM work, there were no cortical thickness changes in the anterior cingulate and supramarginal gyri. In addition this mega-analysis described decreased cortical thickness in regions such as the middle temporal, inferior parietal, precentral, precuneus and lateral occipital regions, for which grey matter volume changes were not evident in previous VBM meta-analyses., ${ }^{3,4}$

The inconsistencies in findings likely reflect, at least partly, the differences in the methodologies of the analyses techniques. Segmentation of structures is performed in fundamentally different ways across the techniques, i.e., FreeSurfer uses a surface-based morphometry approach compared with VBM's voxel-wise registration. ${ }^{39}$ The inconsistencies might also indicate that for some brain regions differences in grey matter volume are not explained by differences in cortical thickness. ${ }^{40}$ Grey matter volume differences as found by VBM can result from (a) a change in cortical thickness in the absence of surface area changes, (b) a change in surface area in the absence of cortical thickness changes, or (c) changes in both cortical thickness and cortical surface area. ${ }^{40}$ An increase in surface area together with a decrease in cortical thickness might mask the effect when expressed in grey matter volume. Conversely, minor increases or decreases in both surface area and cortical thickness may remain undetected when analysed separately, but might reach significance when combined 
with grey matter volume. Therefore, findings of cortical thickness and grey matter volume are complementary and the combined interpretation of both measures strengthens the conclusions that can be drawn from this data-set.

\section{Comorbid anxiety and depression}

Given that comorbid anxiety and depressive disorders are frequently observed in OCD, ${ }^{41}$ we examined whether the lifetime presence of such disorders would partly explain or influence our main findings of decreased cortical thickness in patients with OCD. In the current study prefrontal involvement was most pronounced in the patients with comorbid anxiety disorders, whereas ventral frontal structures and the hippocampus were most affected in those with comorbid major depressive disorder. Previous work has also shown these regions to be affected in both anxiety and major depressive disorder, perhaps suggesting a shared mechanism underlying emotional and cognitive deficits across disorders. ${ }^{7,42,43}$ In addition, decreased cortical thickness of the inferior frontal regions, insula and dorsomedial prefrontal cortex was found in the OCD group compared with controls when excluding the confounding effects of comorbid anxiety and depression in this sample. This suggests that involvement of these cortical areas is unique to OCD. This is consistent with previous work, ${ }^{42}$ together with findings from the OCD VBM mega-analysis. ${ }^{5}$

\section{Impact of medication}

We examined whether psychotropic medication use has a confounding effect on the changes observed in cortical thickness or subcortical volume. For the prefrontal, temporal and occipital regions there was greater cortical thinning in patients with OCD on medication compared with unmedicated patients. Although it has been suggested that selective serotonin reuptake inhibitors might modulate neuroplasticity in brain regions of people with OCD,${ }^{44}$ these findings show little internal consistency. Thus, the extent to which medication has consistent effects on brain structure remains open for future study. That being said, after excluding patients on medication, the main findings in the inferior and superior frontal, precentral, posterior cingulate and middle temporal regions still held true, indicating that irrespective of medication use, these regions are altered in patients with OCD. Indeed, the findings were somewhat more pronounced when patients on medications were excluded, emphasising the power that mega-analyses have to address confounding variables.

The negative association of lateral occipital thickness with the YBOCS severity score is also a unique finding, together with the increased cortical thickness of this region in patients with OCD that were not on medication. Although not part of the corticostriatal and limbic pathways thought to be affected in OCD, previous volumetric studies have found decreased grey matter volume in the occipital lobe of people with OCD. ${ }^{45,46}$

\section{Findings from the symptom dimensions analysis}

In addition, the finding that different symptom dimensions have distinctive neuroanatomical correlates is consistent with previous work. $^{7,16}$ Similar to our present cortical thickness findings, associations of temporal and occipital regional volumes with the harm/checking and symmetry/order dimensions have been shown before, ${ }^{22}$ as well as positive associations of the sexual/religious dimension and hoarding dimensions with prefrontal and lateral orbitofrontal volumes. ${ }^{46}$ Not all FreeSurfer findings of the symptom dimension analyses are consistent with previous VBM analyses, ${ }^{22,47}$ possibly as a result of clinical heterogeneity of participants and differences in quantification of the dimension variables for each study. For example, in this study, symptom dimensions were defined in a binary manner as absent or present for each participant, whereas the above-mentioned studies have correlated the dimension scores with grey matter volumes. Our seemingly incongruent findings of a positive association between cortical thickness and specific dimensions coupled with decreased cortical thickness in patients may reflect these methodological problems in measuring symptom dimensions. In contrast to a considerably clear image of the general structural brain changes in OCD, there is arguably a paucity of literature to support a detailed model of the underlying neuroanatomy of each particular symptom dimension and therefore replication of these findings is warranted. Also, because of the limitations of the clinical data-set, a cluster analysis of symptom dimensions was not possible. ${ }^{48}$ Conclusions drawn from this analysis about the neurocircuitry of symptom dimensions must be tentative.

\section{Strengths and limitations}

The benefit of a large multisite study such as this is the greater sample size, which decreases the likelihood of type I and type II errors. Another benefit is the congruency of preprocessing and analytical methods in a mega-analysis compared with metaanalyses. Sample size and other clinical confounders such as comorbid anxiety/major depressive disorder and medication use have limited previous investigations of cortical thickness in OCD. By performing an analysis in this large group, these clinical confounders can be controlled for more easily.

The present study also has some important limitations. These include the potential confounders of scan sequence and other site differences. Therefore, variance in scan sequence was controlled for in the main and regression analyses. The measurement of symptom dimension scores was based on the symptom checklist of the YBOCS and was perhaps suboptimal because of variance in use of the instrument across the participating centres. Although the strength of this study is that clinical confounders could be controlled for with a stepwise regression and participant exclusion, it is still difficult to fully disentangle the confounding effects of medication and comorbidity. Future studies should employ instruments specifically designed and validated for this purpose. In addition, our group $\times$ age findings are weakened by the cross-sectional design of the study. Future longitudinal studies of brain imaging in OCD will help shed light on the neurodevelopmental aspects of this disorder. This involves both the altered neurodevelopment before the onset of the disease and the disease-related and treatment-related neuroplastic changes during the course of the disease. Also, we performed this analysis on $1.5 \mathrm{~T}$ brain scans, which do have inherent resolution limitations compared with $3 \mathrm{~T}$ MRI. Further work, using longitudinal designs, $3 \mathrm{~T}$ MRI and incorporating genetic and environmental variables, will be useful in understanding the precise mechanisms underlying the structural abnormalities preceding the onset of the disease and that occur during the course of the disease.

In summary, this is the largest cortical thickness and subcortical volume study of OCD to date. The results support the importance of structural abnormalities in fronto-striatal, fronto-parietal and limbic circuits in the pathophysiology of OCD. There is notable consistency of the present FreeSurfer findings with previous VBM metaanalyses and cortical thickness findings with regard to inferior frontal gyrus/operculum and superior frontal gyrus/dorsomedial prefrontal cortex, suggesting that cortical thinning as well as grey matter volume abnormalities could perhaps relate to a similar pathological process underlying the disorder. 
Jean-Paul Fouche, MSc, Department of Psychiatry and Mental Health, University of Cape Town, Cape Town, South Africa; Stefan du Plessis, MD, Department of Psychiatry, University of Stellenbosch, Cape Town, South Africa; Coenie Hattingh MSc, Department of Psychiatry and Mental Health, University of Cape Town, Cape Town, South Africa; Annerine Roos, PhD, MRC Unit on Anxiety \& Stress Disorders, Department of Psychiatry, University of Stellenbosch, Cape Town, South Africa; Christine Lochner, PhD, MRC Unit on Anxiety \& Stress Disorders, Department of Psychiatry, University of Stellenbosch, Cape Town, South Africa; Carles Soriano-Mas, PhD, Department of Psychiatry, Bellvitge University Hospital-IDIBELL, Barcelona, Spain, Carlos III Health Institute, Centro de Investigación Biomédica en Red de Salud Mental (CIBERSAM), Spain, and Department of Psychobiology and Methodology in Health Sciences, Universitat Autònoma de Barcelona, Spain: Joao R. Sato, PhD, Center of Mathematics, Computation and Cognition, Universidade Federal do ABC, Santo Andre, Brazil and Laboratório Interdisciplinar de Neurociências Clínicas, Department of Psychiatry, Universidade Federal de São Paulo, São Paulo, Brazil; Takashi Nakamae, MD, PhD, Department of Psychiatry, Graduate School of Medical Science, Kyoto Prefectural University of Medicine, Kyoto, Japan; Seiji Nishida, MD, PhD, Department of Psychiatry, Graduate School of Medical Science, Kyoto Prefectural University of Medicine, Kyoto, Japan; Jun Soo Kwon, MD, PhD, Department of Psychiatry, Seoul National University College of Medicine, Seoul, Republic of Korea and Institute of Human Behavioral Medicine, SNU-MRC, Seoul, Republic of Korea and Department of Brain and Cognitive Sciences, College of Natura Sciences, Seoul National University, Seoul, Republic of Korea; Wi Hoon Jung, PhD, Department of Psychiatry, Seoul National University College of Medicine, Seoul, Republic of Korea; David Mataix-Cols, PhD, Department of Clinical Neuroscience, Karolinska Institute, Stockholm, Sweden; Marcelo Q. Hoexter, MD, PhD, Laboratório Interdisciplinar de Neurociências Clínicas, Department of Psychiatry, Universidade Federal de São Paulo, São Paulo, Brazil and Department \& Institute of Psychiatry, University of Sao Paulo Medical School, Sao Paulo, Brazil; Pino Alonso, MD, PhD, Department of Psychiatry, Bellvitge University Hospital-IDIBELL, Barcelona, Spain and Carlos III Health Institute, Centro de Investigación Biomédica en Red de Salud Menta (CIBERSAM), Spain, and Department of Clinical Sciences, Faculty of Medicine, University of Barcelona, Spain; Stella J. de Wit, MD, Department of Psychiatry, VU University Medical Centre, Amsterdam, The Netherlands; Dick J. Veltman, MD, PhD, Department of Psychiatry, VU University Medical Centre, Amsterdam, The Netherlands; Dan J. Stein, MD, PhD, Department of Psychiatry and Mental Health, University of Cape Town, Cape Town, South Africa and MRC Unit on Anxiety \& Stres Disorders, Department of Psychiatry, University of Stellenbosch, Cape Town, South Africa; Odile A. van den Heuvel, MD, PhD, Department of Psychiatry, VU University Medical Centre, Amsterdam, The Netherlands, Department of Anatomy \& Neurosciences, VU University Medical Center, Amsterdam, The Netherlands

Correspondence: Jean-Paul Fouche, Department of Psychiatry and Mental Health, University of Cape Town, Faculty of Health Sciences, Anzio Road Observatory, 7935, South Africa. Email: FCHJEA002@myuct.ac.za

\section{Funding}

Supported by the Dutch Organization for Scientific Research (NWO) (grants 912-02-050, 907-00-012, 940-37- 018 and 916.86.038); the Carlos III Health Institute (PI09/01331 PI10/01753, PI10/01003, CP10/00604, PI13/01958 and CIBER-CB06/03/0034); the Agency for Administration of University and Research (AGAUR, Barcelona; 2009SGR1554); a 'Migue Servet' contract from the Carlos III Health Institute (CP10/00604) to C.S.-M.; Ministry of Education, Culture, Sports, Science, and Technology (Japan) Grants-in-Aid for Young Scientists to J.N. (member of the OCD Brain Imaging Consortium, 23591724) and to T.N. (24791223); Wellcome Trust project grant 064846; a grant from the Foundation for the Support of Research in the State of São Paulo (FAPESP) to E.C.M. (member of for the Support of Research in the State of São Paulo (FAPESP) to E.C.M. (member of
the OCD Brain Imaging Consortium, 2011/21357-9); a FAPESP scholarship to M.Q.H. (2005/ 04206-6); and a National Research Foundation of Korea grant funded by the Korean government (Ministry of Education, Science, and Technology, 2012-0005150).

\section{Acknowledgements}

We would like to thank the CSIR Centre for High Performance Computing at Rosebank Cape Town, South Africa for providing the supercomputing facilities to perform Freesurfer image processing and analysis. We would also like to thank all the authors who contributed to this study as well as the members of the OCD Brain Imaging Consortium.

\section{References}

1 Fontenelle LF, Mendlowicz MV, Versiani M. The descriptive epidemiology of obsessive-compulsive disorder. Prog Neuropsychopharmacol Biol Psychiatry 2006; 30: 327-37

2 Hollander E, Stein DJ, Fineberg NA, Marteau F, Legault M. Quality of life outcomes in patients with obsessive-compulsive disorder: relationship to treatment response and symptom relapse. J Clin Psychiatry 2010; 71: 784-92.

3 Rotge J-Y, Guehl D, Dilharreguy B, Tignol J, Bioulac B, Allard M, et al. Meta-analysis of brain volume changes in obsessive-compulsive disorder. Biol Psychiatry 2009; 65: 75-83.
4 Rotge J-Y, Langbour N, Guehl D, Bioulac B, Jaafari N, Allard M, et al. Gray matter alterations in obsessive-compulsive disorder: an anatomic likelihood estimation meta-analysis. Neuropsychopharmacology 2010; 35: 686-91.

5 de Wit SJ, Alonso P, Schweren L, Mataix-Cols D, Lochner C, Menchón JM, et al. Multicenter voxel-based morphometry mega-analysis of structural brain scans in obsessive-compulsive disorder. Am J Psychiatry 2014; 171: 340-9.

6 Radua J, Mataix-Cols D. Voxel-wise meta-analysis of grey matter changes in obsessive-compulsive disorder. Br J Psychiatry 2009; 195: 393-402.

7 Radua J, van den Heuvel OA, Surguladze S, Mataix-Cols D. Meta-analytical comparison of voxel-based morphometry studies in obsessive-compulsive disorder vs other anxiety disorders. Arch Gen Psychiatry 2010; 67: 701-11.

8 Tops M, Boksem MAS. A potential role of the inferior frontal gyrus and anterior insula in cognitive control, brain rhythms, and event-related potentials. Front Psychol 2011; 2: 330

9 Dale AM, Fischl B, Sereno MI. Cortical surface-based analysis. I. Segmentation and surface reconstruction. Neuroimage 1999; 9: 179-94.

10 Wonderlick JS, Ziegler DA, Hosseini-Varnamkhasti P, Locascio JJ, Bakkour A, van der Kouwe A, et al. Reliability of MRI-derived cortical and subcortical morphometric measures: effects of pulse sequence, voxel geometry, and parallel imaging. Neuroimage 2009; 44: 1324-33.

11 Shin Y-W, Yoo SY, Lee JK, Ha TH, Lee KJ, Lee JM, et al. Cortical thinning in obsessive compulsive disorder. Hum Brain Mapp 2007: 28: 1128-35.

12 Kühn S, Kaufmann C, Simon D, Endrass T, Gallinat J, Kathmann N. Reduced thickness of anterior cingulate cortex in obsessive-compulsive disorder. Cortex 2013; 49: 2178-85

13 Venkatasubramanian G, Zutshi A, Jindal S, Srikanth SG, Kovoor JME, Kuma $\mathrm{JK}$, et al. Comprehensive evaluation of cortical structure abnormalities in drug-naïve, adult patients with obsessive-compulsive disorder: a surfacebased morphometry study. J Psychiatr Res 2012; 46: 1161-8.

14 Fullana MA, Cardoner $\mathrm{N}$, Alonso $\mathrm{P}$, Subirà $\mathrm{M}$, López-Solà $\mathrm{C}$, Pujol J, et al. Brain regions related to fear extinction in obsessive-compulsive disorder and its relation to exposure therapy outcome: a morphometric study. Psychol Med 2014; 44: 845-56.

15 Nakamae T, Narumoto J, Sakai Y, Nishida S, Yamada K, Kubota M, et al. Reduced cortical thickness in non-medicated patients with obsessivecompulsive disorder. Prog Neuropsychopharmacol Biol Psychiatry 2012; 37 90-5.

16 Narayan VM, Narr KL, Phillips OR, Thompson PM, Toga AW, Szeszko PR. Greater regional cortical gray matter thickness in obsessive-compulsive disorder. Neuroreport 2008; 19: 1551-5.

17 Fan Q, Palaniyappan L, Tan L, Wang J, Wang X, Li C, et al. Surface anatomical profile of the cerebral cortex in obsessive-compulsive disorder: a study of cortical thickness, folding and surface area. Psychol Med 2013; 43: 1081-91.

18 Goodman WK, Price LH, Rasmussen SA, Mazure C, Fleischmann RL, Hill CL, et al. The Yale-Brown Obsessive Compulsive Scale. I. Development, use, and reliability. Arch Gen Psychiatry 1989; 46: 1006-11.

19 American Psychiatric Association. Diagnostic and Statistical Manual of Mental Disorder (4th edn) (DSM-IV). APA, 1994.

20 Fischl B, Dale AM. Measuring the thickness of the human cerebral cortex from magnetic resonance images. Proc Natl Acad Sci USA 2000; 97 $11050-5$

21 Mathalon DH, Sullivan EV, Rawles JM, Pfefferbaum A. Correction for head size in brain-imaging measurements. Psychiatry Res 1993; 50: 121-39.

22 van den Heuvel OA, Remijnse PL, Mataix-Cols D, Vrenken $\mathrm{H}$, Groenewegen $\mathrm{HJ}$, Uylings HBM, et al. The major symptom dimensions of obsessivecompulsive disorder are mediated by partially distinct neural systems. Brain 2009; 132: 853-68.

23 Pujol J, Soriano-Mas C, Alonso P, Cardoner N, Menchón JM, Deus J, et al. Mapping structural brain alterations in obsessive-compulsive disorder. Arch Gen Psychiatry 2004; 61: 720-30.

24 Yoo SY, Roh M-S, Choi J-S, Kang D-H, Ha TH, Lee JM, et al. Voxel-based morphometry study of gray matter abnormalities in obsessive-compulsive disorder. J Korean Med Sci 2008; 23: 24-30.

25 Li P, Li S, Dong Z, Luo J, Han H, Xiong H, et al. Altered resting state functional connectivity patterns of the anterior prefrontal cortex in obsessivecompulsive disorder. Neuroreport 2012; 23: 681-6.

26 Kim S-G, Jung WH, Kim SN, Jang JH, Kwon JS. Disparity between dorsal and ventral networks in patients with obsessive-compulsive disorder: evidence revealed by graph theoretical analysis based on cortical thickness from MRI. Front Hum Neurosci 2013; 7: 302.

27 Bannon S, Gonsalvez CJ, Croft RJ, Boyce PM. Executive functions in obsessive-compulsive disorder: state or trait deficits? Aust NZ J Psychiatry 2006; 40: 1031-8 
28 Penadés R, Catalán R, Rubia K, Andrés S, Salamero M, Gastó C. Impaired response inhibition in obsessive compulsive disorder. Eur Psychiatry 2007; 22: $404-10$.

29 van den Heuvel OA, Veltman DJ, Groenewegen HJ, Cath DC, van Balkom AJLM, van Hartskamp J, et al. Frontal-striatal dysfunction during planning in obsessive-compulsive disorder. Arch Gen Psychiatry 2005; 62: 301-9.

30 Chamberlain SR, Fineberg NA, Blackwell AD, Clark L, Robbins TW, Sahakian BJ. A neuropsychological comparison of obsessive-compulsive disorder and trichotillomania. Neuropsychologia 2007; 45: 654-62.

31 Menzies L, Chamberlain SR, Laird AR, Thelen SM, Sahakian BJ, Bullmore ET. Integrating evidence from neuroimaging and neuropsychological studies of obsessive-compulsive disorder: the orbitofronto-striatal model revisited. Neurosci Biobehav Rev 2008; 32: 525-49.

32 Stern ER, Fitzgerald KD, Welsh RC, Abelson JL, Taylor SF. Resting-state functional connectivity between fronto-parietal and default mode networks in obsessive-compulsive disorder. PLOS One 2012; 7: e36356.

33 Zhang T, Wang J, Yang Y, Wu Q, Li B, Chen L, et al. Abnormal small-world architecture of top-down control networks in obsessive-compulsive disorder. J Psychiatry Neurosci 2011; 36: 23-31.

34 Meunier D, Ersche KD, Craig KJ, Fornito A, Merlo-Pich E, Fineberg NA, et al. Brain functional connectivity in stimulant drug dependence and obsessivecompulsive disorder. Neuroimage 2012; 59: 1461-8.

35 Fischl B, Salat DH, Busa E, Albert M, Dieterich M, Haselgrove $C$, et al. Whole brain segmentation: automated labeling of neuroanatomical structures in the human brain. Neuron 2002; 33: 341-55.

36 Zhou Y, Zambelli J, Heredia G. SU-E-I-70: Comparison of brain volume quantification with NeuroQuant \& FreeSurfer. Med Phys 2015; 42: 3258.

37 Sowell ER, Thompson PM, Toga AW. Mapping changes in the human cortex throughout the span of life. Neuroscientist 2004; 10: 372-92.

38 Grieve SM, Clark CR, Williams LM, Peduto AJ, Gordon E. Preservation of limbic and paralimbic structures in aging. Hum Brain Mapp 2005; 25 391-401.

39 Clarkson MJ, Cardoso MJ, Ridgway GR, Modat M, Leung KK, Rohrer JD, et al. A comparison of voxel and surface based cortical thickness estimation methods. Neuroimage 2011; 57: 856-65.
40 Palaniyappan L, Liddle PF. Differential effects of surface area, gyrification and cortical thickness on voxel based morphometric deficits in schizophrenia. Neuroimage 2012; 60: 693-9.

41 Ruscio AM, Stein DJ, Chiu WT, Kessler RC. The epidemiology of obsessivecompulsive disorder in the National Comorbidity Survey Replication. $\mathrm{Mol}$ Psychiatry 2010; 15: 53-63.

42 van Tol M-J, van Der Wee NJA, van den Heuvel OA, Nielen MMA, Demenescu LR, Aleman A, et al. Regional brain volume in depression and anxiety disorders. Arch Gen Psychiatry 2010; 67: 1002-11.

43 Cardoner N, Soriano-Mas C, Pujol J, Alonso P, Harrison BJ, Deus J, et al. Brain structural correlates of depressive comorbidity in obsessive-compulsive disorder. Neuroimage 2007; 38: 413-21.

44 Hoexter MQ, de Souza Duran FL, D'Alcante CC, Dougherty DD, Shavitt RG, Lopes AC, et al. Gray matter volumes in obsessive-compulsive disorder before and after fluoxetine or cognitive-behavior therapy: a randomized clinical trial. Neuropsychopharmacology 2012; 37: 734-45.

45 Togao O, Yoshiura T, Nakao T, Nabeyama M, Sanematsu H, Nakagawa A, et al. Regional gray and white matter volume abnormalities in obsessivecompulsive disorder: a voxel-based morphometry study. Psychiatry Res 2010; 184: 29-37.

46 Kim J-J, Lee MC, Kim J, Kim IY, Kim SI, Han MH, et al. Grey matter abnormalities in obsessive-compulsive disorder: statistical parametric mapping of segmented magnetic resonance images. Br J Psychiatry 2001; 179: $330-4$

47 Alvarenga PG, do Rosário MC, Batistuzzo MC, Diniz JB, Shavitt RG, Duran FLS, et al. Obsessive-compulsive symptom dimensions correlate to specific gray matter volumes in treatment-naïve patients. J Psychiatr Res 2012; 46 1635-42.

48 Lochner C, Hemmings SMJ, Kinnear CJ, Nel D, Hemmings SMJ, Seedat S, et al. Cluster analysis of obsessive-compulsive symptomatology: identifying obsessive-compulsive disorder subtypes. ISr J Psychiatry Relat Sci 2008; 45 164-76. 